\title{
Studies on the Rapid Identification of Gram-negative Rods in Fresh Fish, with Special Reference to the Problems on the Corlett's Replica Plating Method
}

(Received January 27, 1969)

\author{
Toshiharu KAWABATA*1, Tsuneo KOZIMA*2 and Yasuhiko SAKAGUCHI*2 \\ ( ${ }^{* 1}$ Department of Food Research, National Institute of Health, \\ 10-35, Kamiosaki 2-chome, Shinagawa-ku, Tokyo, Japan; \\ *2Department of Food Technology, Tokyo University of Fisheries, \\ 5-7, Konan 4-chome, Minato-ku, Tokyo, Japan)
}

\begin{abstract}
In order to get more simplified and rapid method for the identification of microorganisms, especially for Gram-negative rods in various sea foods, the method proposed by Corlett et al. was repeated in the present experiment using 39 reference cultures of several genera, and 754 cultures of the isolates from fish which had been identified according to the method by Kawabata et al.

Results obtained may be summarized as follows. In addition, some partial modifications on the replica plating method are being proposed:

Good coincidence was observed in the test organisms belonging to Pseudomonas group II, Flavobacterium and coliform, of both reference cultures and the isolates.

Several reference and isolated cultures belonging to Pseudomonas group I species, however, were identified as Pseudomonas group II according to the original method by Corlett et al. This was found to be resulted from the use of an improper basal medium (TPN) on which the test organisms failed to produce green fluorescent pigments. The difficulty or defect in the original method could be solved by using the medium B of King et al. instead of TPN.

We also encountered with difficulty in identifying genus Achromobacter; among the test organisms consisting of 7 reference cultures and 38 isolates of Achromobacter species, only 8 species could be identified as they are, and the remainders were unidentified according to the key proposed by Corlett et al. These results strongly suggest the need for further studies so as to solve the defect in identifying genus Achromobacter.
\end{abstract}

\section{Introduction}

In many fields of microbiology, the Bergey's Manual of Determinative Bacteriology is widely used as a standard reference book for the identification of microorganisms, especially the book may give many useful informations on classifying bacteria to species level.

However, when we want to know the types of spoilage, to determine the storage capacity or to evaluate the potential hazards, quantitative determination of the microflora of a food is necessary, and for this purpose, it would be sufficient to identify isolates at generic levels.

Several identification protocols for the bacterial

*1 河端俊治 (国立予防衛生研究所)

*2 小嶋秩夫, 坂口靖彦 (東京水産大学) isolates in various foods at generic level have been proposed by Maslovsky et al., ${ }^{11}$ Hendrie et al., ${ }^{21}$ Shewan et al. ${ }^{3 \prime}$ and Kanazas. ${ }^{4 !}$

In our laboratory, methods for the identification of certain Gram-negative organisms in various sea foods have been the subject of study. And recently, an improved determinative scheme shown in Fig. 1 is being compared with aforementioned schemes.

However, the methods used are often tedious and time-consuming and they require special skill in identifying bacteria even at generic levels. In the consideration of quantitative evaluation of microbial flora in foods, establishment of a more simplified and rapid identification method is desired.

Recently, a rapid identification method using 


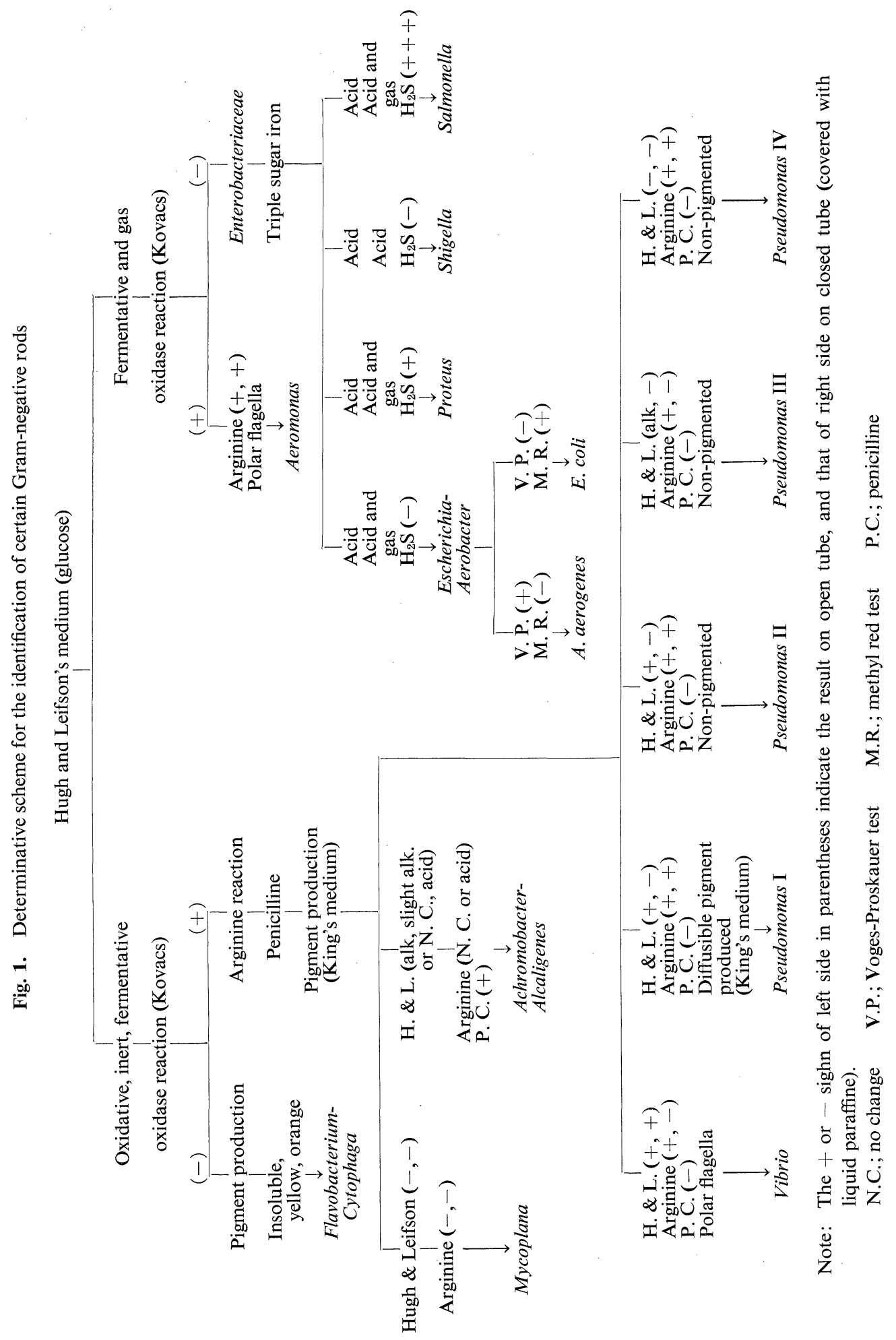


replica plating and computer analysis has been proposed by Corlett et al. ${ }^{51} \quad$ The principle of their method was based on responses of microorganisms on several antibiotic and selective media together with tests for morphological characters. This method was also characterized by using a computer for analysing data. According to Corlett et al., ${ }^{5}$ ' the examination may fit for a large number of microbial isolates with simplicity.

It is well known that in such a food as fish various Gram-negative rods and certain Grampositive cocci are dominating to cause spoilage of these foods, while very low in the distribution of Gram-positive rods.

Since cocci may be classified to genera by morphological characteristics or by simple tests, it is Gram-negative rods which must be taken into consideration of a systematic identification scheme.

In this study, we have attempted to compare the results obtained by Corlett's replica plating method and those by the identification protocol shown in Fig. 1 in order to get basic knowledges for establishing a more rapid identification method of Gram-negative rods in fish.

\section{Materials and Methods}

1. Outline of Corlett's replica plating method, and methods for the present experiment:

The original replica plating method ${ }^{5) \sim 71}$ has been divided into the following four steps;

1) isolation of bacteria to be tested;2) inoculation of isolates on master plate; 3 ) replica plating; and 4) analysis of data.

Firstly, food is homogenized, diluted and plated according to the conventional procedures. Instead of using pour plates, however, spreadplating was used to facilitate later isolation of the colony. The number of bacteria may be $10^{6} / \mathrm{g}$ at initial stage of spoilage and $10^{8} / \mathrm{g}$ at final stage, which may be used as a mark of dilution.

All isolated colonies were picked by sterile toothpicks and transferred to the master plates.

Microorganisms were inoculated on a master plate according to prearranged position and spacing. In the present study, we used Petri dishes of $9 \mathrm{~cm}$ in diameter and each master plate could accomodate 19 isolates.

Replica plating was made from a master plate to a series of daughter plates that contained several differential or selective agents. The tool needed for replication, originally reported by Lederberg and Lederberg ${ }^{81}$ was adapted to the present study.

The daughter plates consisted of 2 basal medium plates and 7 antibiotic media, viz., penicilline, tylosin, vancomycin, streptomycin-chloramphenicol mixture, neomycin and colistin, and following 3 different media, SS medium, staphylococcus 110 and potato dextrose agar.

The first basal medium plate was used to observe the pigmentation of colonies and production of green fluorescent, pigments under ultraviolet, and the determination of the Gram reaction. The last plate of a replication series served to verify the successful transfer of all colonies to the preceding plates.

Daughter plates were incubated at $30^{\circ} \mathrm{C}$ for $24 \mathrm{hrs}$ or $48 \mathrm{hrs}$ and growth reactions were recorded as identification key. Corlett et al. ${ }^{5}$ employed 10 differential and selective media for identification purposes, and they expressed the criteria based on the responses appeared on 12 daughter plates in such simple code as 0,1 and 2 .

Since the present study was aimed to compare the results obtained by our method (Fig. 1) with those by Corlett's replica plating, a number of reference cultures and isolates from various fish samples in the several experiments conducted at the Department of Food Research, National Institute of Health were used for the present study. Therefore, we eliminated the isolation step from fish samples, initiating experiments from the step of inoculation of Gram-negative rods of both reference cultures and isolates on master plates. Moreover nitrofrazone (NF) and oxytetracycline (OTC) plates were employed in the present study in addition to the Corlett's antibiotic media plates, while potato dextorose agar for detecting molds and yeast in food was omitted in our study.

\section{Reference cultures used:}

Pseudomonas fluorescens (IAM 1201, IAM 1006, AHU 1143, NEER B-10), Ps. putida (IAM 1506), Ps. rubescens (IAM 1510), $P_{s}$. mildenbergii (IAM 1505), Ps. schuylkilliensis (IAM 1008), Ps. fragi (IAM 1650), Ps. synxantha (IFO 3912), Ps. aeruginosa (IAM 1007).

Achromobacter cycloclastes (IAM 1013), A. halophilus (AHU 1333), A. guttatus (NCIB 9405), A. lacticum (NCIB 8209), A. sp. (NCMB 29, NCMB 132), A. candicans, A. stenohalis 
AHU 1336).

Flavobacterium sulfureum var. miyamizu (IAM 1252), F. suaveolens (IFO 3752), F. aquatile (IFO 3772).

Escherichia coli (0-1 and 0-2, IAM 1239).

Klebsiella (6552, 6553 and 6555).

Aerobacter cloacae (IAM 1134).

These reference cultures were kindly provided by the Institute of Applied Microbiology, the University of Tokyo, Tokyo; Institute of Fermentation, Osaka; Faculty of Agriculture, Hokkaido University, Sapporo, Hokkaido; Institute of Food Microbiology, Chiba University, Chiba; and Laboratory of Microbiology, Tokyo University of Fisheries, Tokyo.

\section{Isolates tested:}

Pseudomonas group I, 247 strains; group II, 408 strains; group III-IV, 36 strains.

Achromobacter, 38 strains (may be including Cytophaga)

Flavobacterium, 7 strains.

Coliform, 18 strains.

All the isolates were obtained from fish sources in various experiments, and identified according to the determinative protocol shown in Fig. 1.

\section{Basal medium:}

The basal medium employed in the present study was quite identical with that employed by Corlett et al. ${ }^{51}$ This medium (TPN) was composed of $0.5 \%$ peptone (Difco), $0.5 \%$ Tryptone (Difco), $0.25 \%$ yeast extract (Difco), $0.5 \% \mathrm{NaCl}$ (reagent grade), $0.1 \%$ glucose and $2 \%$ agar (Difco) in distilled water.

Antibiotic media for differential or selective purpose: Each antibiotic solution at respective concentration listed below was added to the basal medium; penicilline G potassium, $\quad 3 \mathrm{IU} / \mathrm{ml}$ (PEN) tylosin lactate, $10 \mu \mathrm{g} / \mathrm{ml}$ (TY) vancomycin sulfate, streptomycin sulfate, streptomycin sulfate, $10 \mu \mathrm{g} / \mathrm{ml}(\mathrm{VAN})$ $10 \mu \mathrm{g} / \mathrm{ml}$ (STR) $10 \mu \mathrm{g} / \mathrm{ml}+$ chloramphenicol, $10 \mu \mathrm{g} / \mathrm{ml}$ (S-C)

neomycin (fradiomycin sulfate), $75 \mu \mathrm{g} / \mathrm{ml}$ (NEO) colistin sulfate, $100 \mu \mathrm{g} / \mathrm{ml}$ (CS)

oxytetracyline hydrochloride, $5 \mu \mathrm{g} / \mathrm{ml}$ (OTC) nitrofurazone, $50 \mu \mathrm{g} / \mathrm{ml}(\mathrm{NF})$ Each antibiotic dissolved in distilled water was added to the melted basal medium which had been sterilized and tempered at about $50^{\circ} \mathrm{C}$. To make free from bacteria, each test antibiotic solution was filtered through a Millipore filter (pore size, $0.22 \mu$ ).

Non antibiotic media used for differential or selective purpose:

\section{SS agar (Eiken)}

staphylococcus medium, 110 (Eiken)

The tool for replica plating:

The tool was made of wooden plate, the diameter of which was smaller than the inside diameter of a Petri dish $(9 \mathrm{~cm})$ and an aluminum plate was placed on it. The surface of a tool was covered with a sheet of velveteen.

We found that a wooden plate often bent after autoclaving which made the successful transfer of all colonies on a daughter plate impossible. To eliminate this kind of trouble, an aluminum circular plate (thickness, $1 \mathrm{~mm}$ ) was placed between velveteen sheet and wooden cylinder surface. After autoclaving the tool, an imprint of the plate was made on the velveteen block and the daughter plates were succesively inoculated from it by gently pressing the velveteen block.

\section{Incubation temperature and period:}

The incubation temperature was selected at $30^{\circ} \mathrm{C}$ to allow the rapid growth of both psychrophiles and mesophiles, and the growth reaction of test organisms was recorded after $24 \mathrm{hrs}$. or $48 \mathrm{hrs}$. of incubation.

\section{The identification protocol for Gram-negative} rods in fish:

All isolates from fish sample were identified at generic levels according to the identification protocol shown in Fig. 1. ${ }^{91}$

3. Growth of Achromobacter and Pseudomonas species on agar plates containing varying concentrations of $\mathrm{NaCl}$ :

Corlett et al..$^{51}$ reported that the staphylococcus 110 medium was chosen as a selective medium for Staphylococcus, Sarcina, Micrococcus and Achromobacter, and the medium was particularly valuable for separating the Achromobacter species from the other Gram-negative rods. The principal mechanism for the selective action of 110 medium has been reported to bo due to its high $\mathrm{NaCl}$ content. ${ }^{101}$ However, our preliminary experiment with some 8 strains of reference cultures of Achromobacter revealed that there existed a variety of resistance 
in the Achromobacter species to $\mathrm{NaCl}$. These facts might strongly suggest that the need for further studies on the growth response of various species of Achromobacter on the staphylococcus 110 medium.

Plate count medium (Difco) containing 0,1 , $3,5,7$ and $9 \%$ of $\mathrm{NaCl}$ were prepared and plated, respectively. Each plate was divided into ten to fifteen sections with a marking pen, on which was smeared each test organism in one area. The plates were incubated at $30^{\circ} \mathrm{C}$ for $48 \mathrm{hrs}$. to observe the growth response of test organisms.

\section{Results and Discussion}

Reference cultures, and isolates from fish samples belonging to various genera, which had been identified by the method shown in Fig. 1 were examined for their growth responses on the selective or differential media used in the Corlett's replica plating method, and results obtained were shown in the following tables; Pseudomonas in Tables 2 and 3, Achromobacter in Tables 4 and 5, Flavobacterium in Table 6 and Coliform in Table 7. As to the results of isolates, however, the number of strains shown in each table is confined within the limit of space.

Table 1 indicates the key for identification of various Gram-negative rods which has been referred from the data of Corlett et al. ${ }^{51}$

\section{Pseudomonas:}

As shown in Table 3, three isolates were identified as Pseudomonas group I species by the replica plating method. However, as can be seen in Table 2 , among the reference cultures of group I, 4 strains out of 8 were identified to be group I, and the remainders were group II. The discrepancy in the identification was principally due to the negative responses of some cultures in producing green fluorescent pigments which was regarded as the most important feature of group I species.

Production of diffusible fluorescent pigments by some Pseudomonas may be enhanced in such medium as King's $\mathrm{B},{ }^{11)}$ and the failure in producing the pigments by some reference cultures might be due to deficiencies of certain components in the basal medium (TPN), nevertheless the addition of peptone to the basal medium has been reported to enhance the production of pigments by some Pseudomonas. $^{5}$ ' Then, 6 reference cultures belonging to Pseudomonas group I, 3 of group II and 1 of group IV, and 7 isolates of group I and 2 of the other groups were inoculated on plates of King's medium $\mathrm{B}$ and Corlett's TPN medium to compare with the production of green fluorescent pigments.

Results obtained were shown in Table 8. Of reference cultures, all species belonging to group I produced clear green fluorescent pigments on the King's medium B in 48 hrs. While on TPN plates,

Table 1. Key for the Identification of Gram-negative Rods in the Replica Plating Method Proposed by Corlett et al. (1965)

\begin{tabular}{|c|c|c|c|c|c|c|c|c|c|c|c|c|c|c|c|c|c|c|c|}
\hline \multirow{2}{*}{ Organisms } & \multicolumn{10}{|c|}{ Growth*1 } & \multicolumn{5}{|c|}{ Pigmentation*2 } & \multicolumn{4}{|c|}{$\begin{array}{l}\text { Morphology*3 } \\
\text { after Gram stain }\end{array}$} \\
\hline & \multicolumn{2}{|c|}{ PEN TY } & \multicolumn{2}{|c|}{ SS VAN } & $\mathrm{S}-\mathrm{C}$ & 110 & STR & \multicolumn{3}{|c|}{ NEO CS PD } & \multirow{2}{*}{$\frac{Y}{0}$} & \multirow{2}{*}{$\begin{array}{l}\mathrm{O} \\
0\end{array}$} & \multirow[t]{2}{*}{$\mathbf{T}$} & \multirow{2}{*}{$\begin{array}{c}\text { WS } \\
0\end{array}$} & \multirow{2}{*}{$\frac{\text { FL }}{0}$} & \multirow{2}{*}{$\begin{array}{l}\mathrm{R} \\
1\end{array}$} & \multicolumn{3}{|c|}{ C OV GR } \\
\hline Coliforms & 1 & 1 & 2 & 1 & & 0 & & & & 0 & & & & & & & 0 & 0 & 0 \\
\hline Pseudomonas, group I & 1 & 1 & 1 & 1 & 1 & 0 & & & & 0 & 0 & 0 & 0 & 1 & 1 & 1 & 0 & 0 & 0 \\
\hline Pseudomonas, group II & 1 & 1 & & 1 & & 0 & & & & 0 & 0 & 0 & 0 & 0 & 0 & 1 & 0 & 0 & 0 \\
\hline $\begin{array}{l}\text { Pseudomonas, group III } \\
\text { and IV }\end{array}$ & & & & & 0 & 0 & 0 & 0 & 0 & 0 & 0 & 0 & 0 & 0 & 0 & 1 & 0 & 0 & 0 \\
\hline \multirow[t]{3}{*}{ Achromobacter } & & 1 & 0 & 1 & 0 & 1 & 0 & 0 & 0 & 0 & 0 & 0 & 0 & 0 & 0 & 1 & 0 & 0 & 0 \\
\hline & & 1 & 0 & 1 & 0 & 1 & 0 & 0 & 0 & 0 & 0 & 0 & 0 & 0 & 0 & 0 & 0 & 1 & 0 \\
\hline & & & & & & & & 0 & 0 & 0 & 0 & 0 & 0 & 0 & 0 & 0 & 0 & 1 & 0 \\
\hline \multirow[t]{3}{*}{ Flavobacterium } & & & 0 & & & 0 & & & & 0 & 1 & 0 & 0 & 0 & 0 & 1 & 0 & 0 & 0 \\
\hline & & & 0 & & & 0 & & & & 0 & 0 & 1 & 0 & 0 & 0 & 1 & 0 & 0 & 0 \\
\hline & & & 0 & & & 0 & & & & 0 & 0 & 0 & 1 & 0 & 0 & 1 & 0 & & 0 \\
\hline
\end{tabular}

Note: *1 1: growth and the other positive reaction 2: two positive response 0 : negative response

*2 Y: yellow; O: orange; $\mathrm{T}$ : tan; WS: water-soluble pigments; FL: ultraviolet fluorescence

*3 $\mathrm{R}$ : rod; $\mathrm{C}$ : coccus; OV: oval or stout rod; GR: Gram reaction 


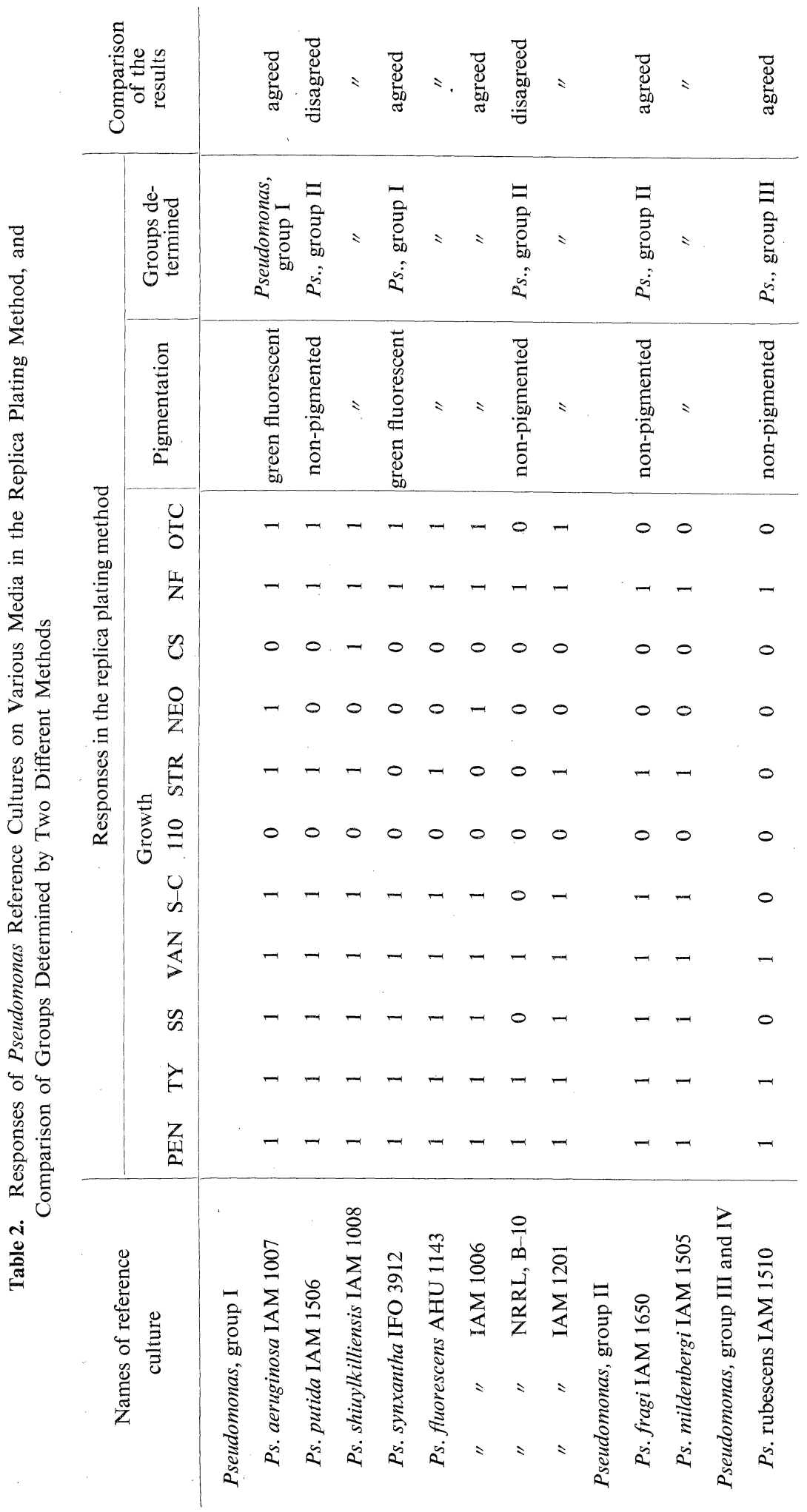




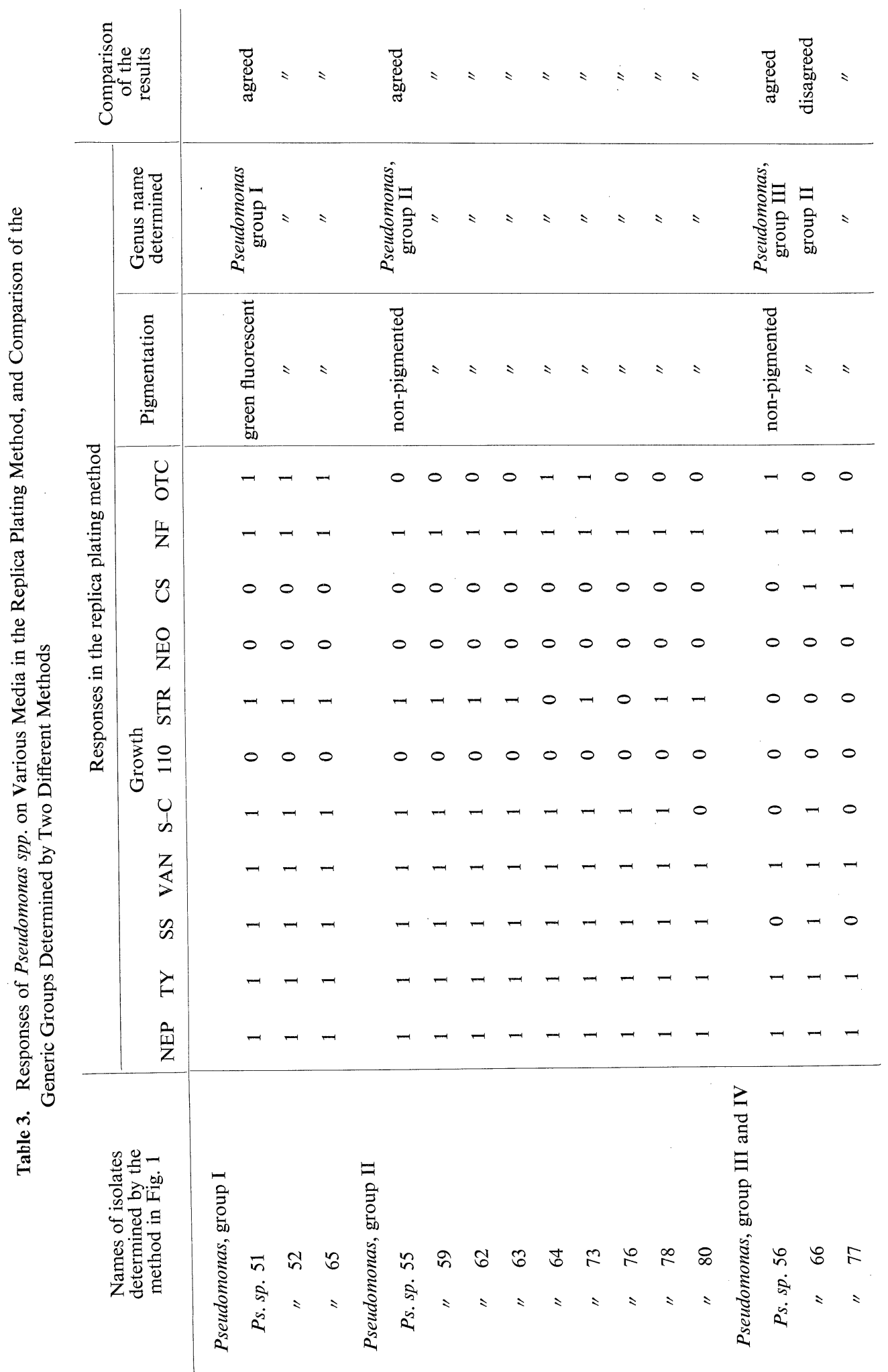




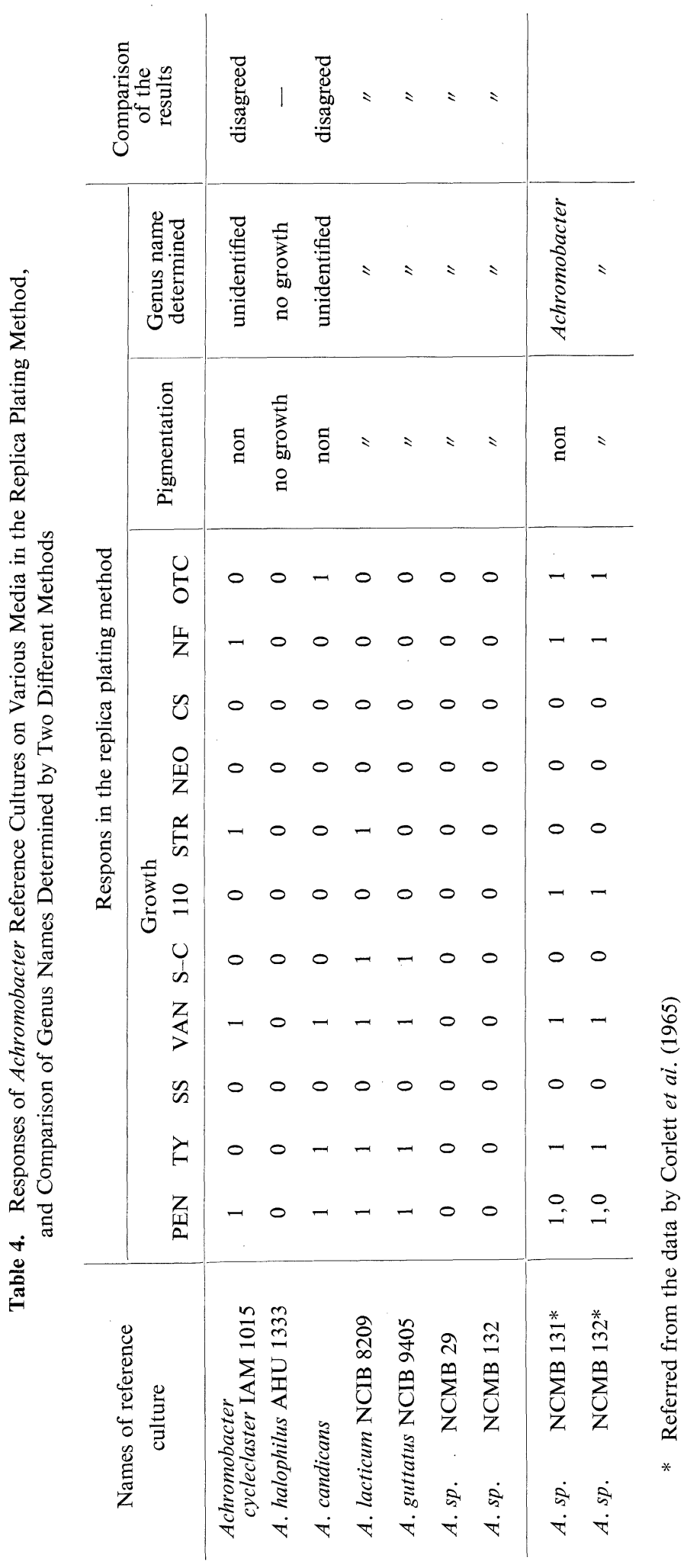




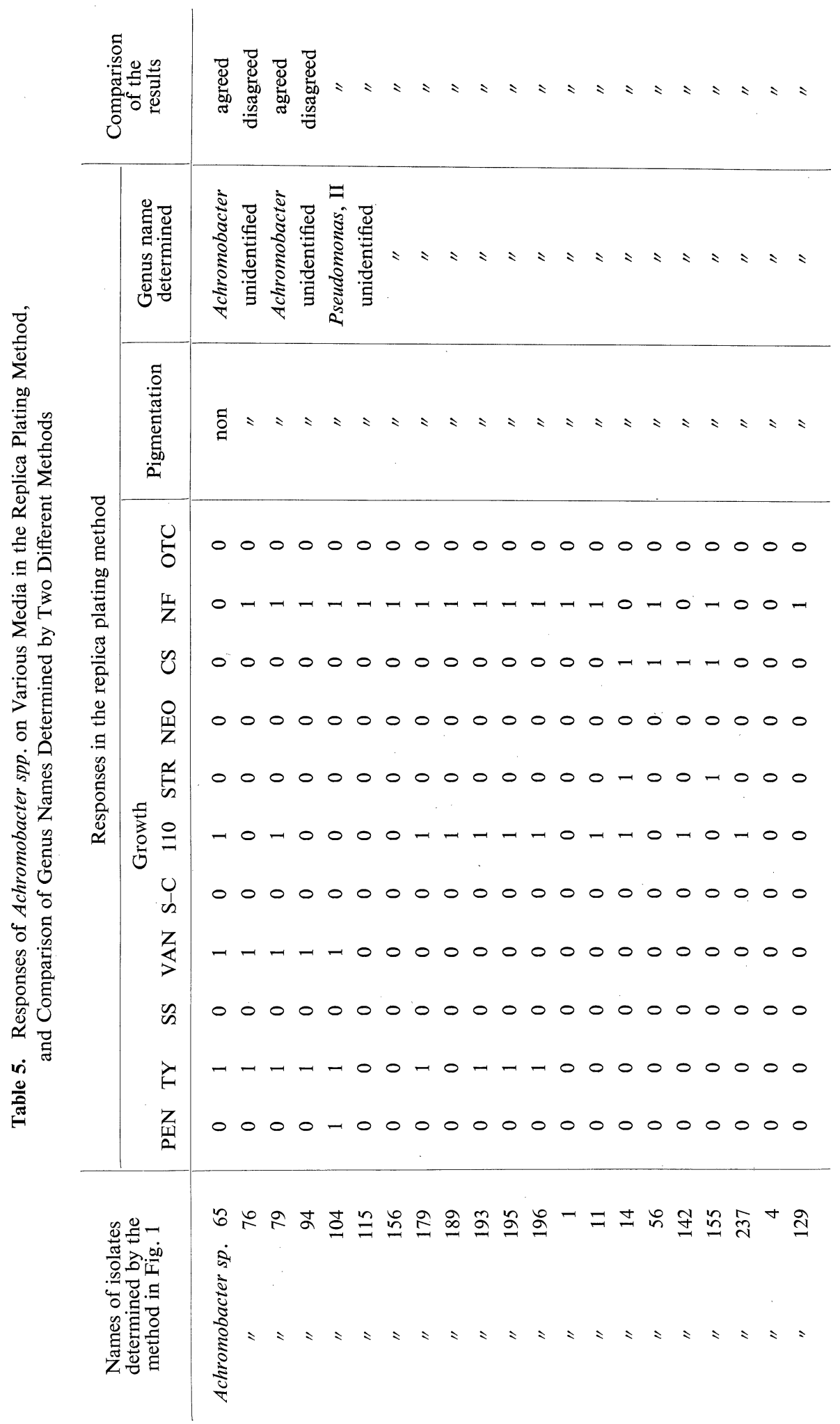


Table 6. Responses of Flavobacterium and Cytophaga on Various Media in the Replica Plating Method, and Comparison of Genus Names Determined by Two Different Methods

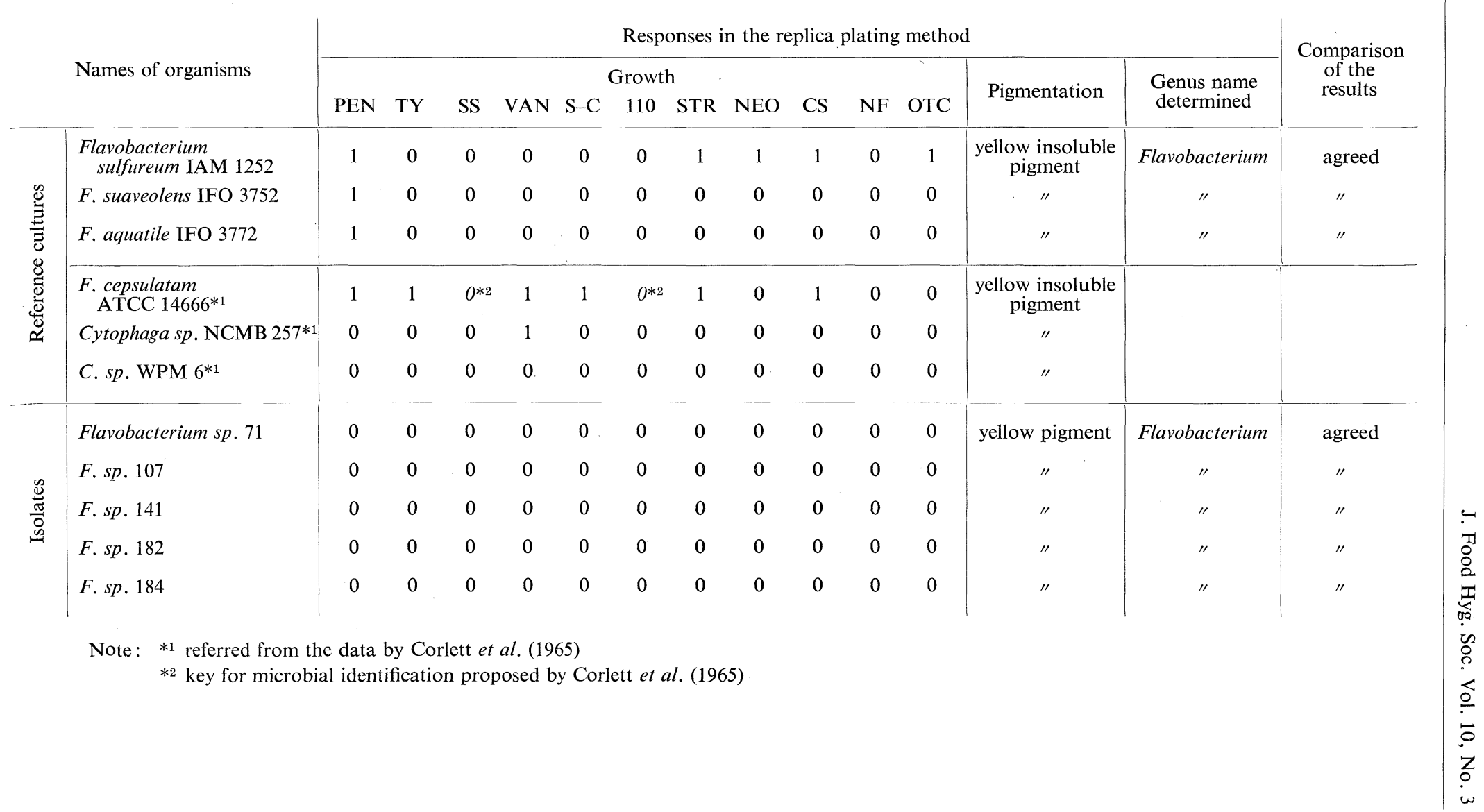




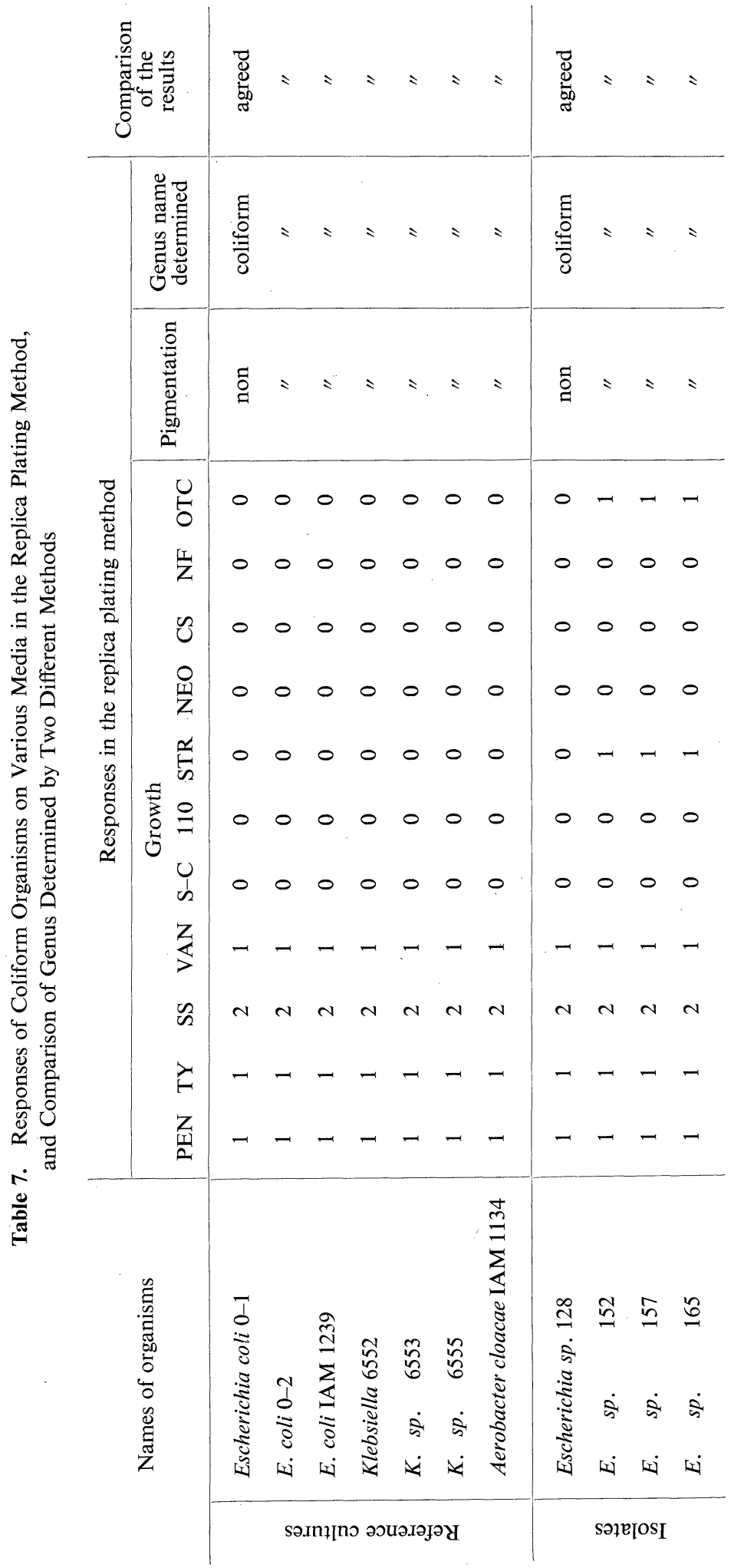


Table 8. Production of Diffusible Green Fluorescence on King's Medium B and Corlett's Basal Medium for Replica Plating (TPN)

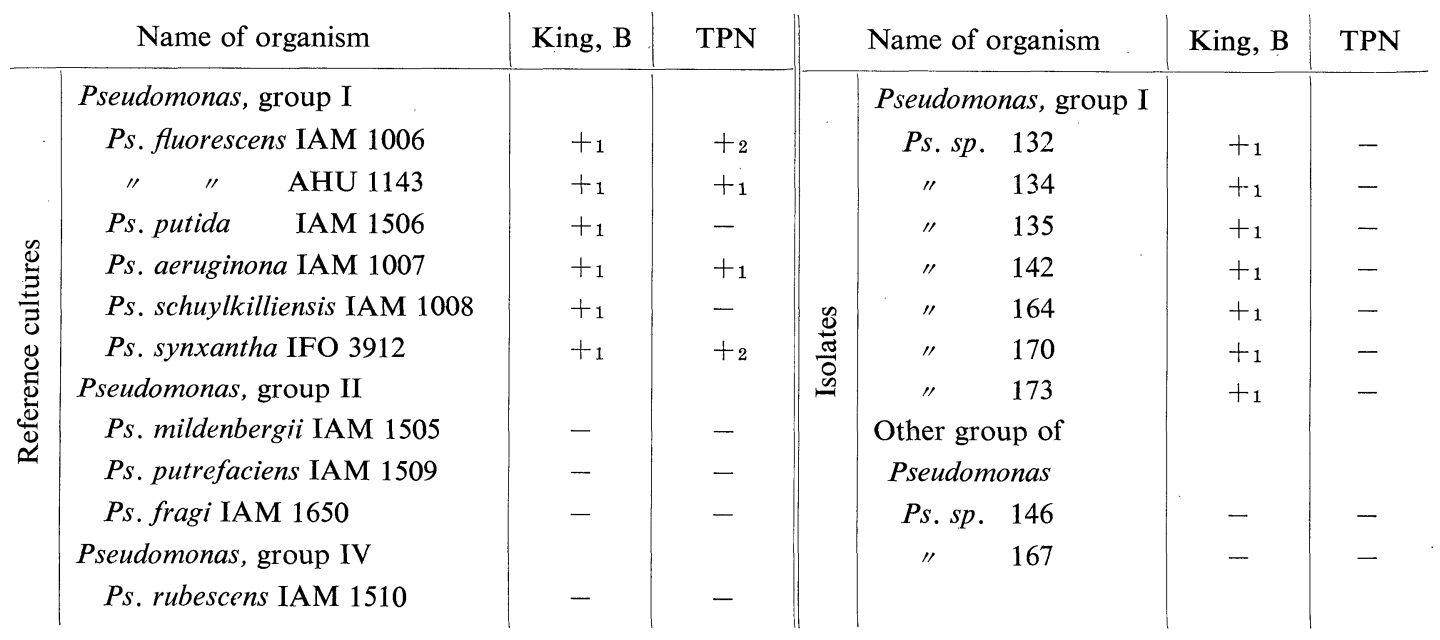

Note: Figures under + sign indicate the period in day required to produce fluorescence

only 4 out of 6 reference cultures of group I produced the pigments. These results coincide with those shown in Table 2. And all the isolates belonging to group I so far tested produced green fluorescent pigments on King's medium B, whereas none produced the pigments on TPN medium.

A marked difference was observed in the ability of producing green fluorescent pigments between King's medium B and TPN medium. The discrepancy in the identification of group I organisms by the replica plating method shown in Table 2 could have resulted from the use of such an improper medium as TPN for the examination of fluorescent pigments, suggesting the necessity of the use of King's medium B instead of TPN as the first plate in the daughter plate series.

Among reference cultures, only Ps. fluorescens (NRRL B-10) did not grow on the SS medium plate, however the other features of the strain were coincident with those of Pseudomonas group I species, especially the ability to produce green fluorescent pigments on King's medium, B, consequently this species could be regarded as an "atypical" Pseudomonas group I.

All Pseudomonas species belonging to group II so far tested in this study were identified as they were by the replica plating method.

Here, a question arises from the key for identifying Pseudomonas group III-IV. Corlett et $a l .{ }^{5}{ }^{\prime}$ reported that Pseudomonas group III-IV species were sensitive to all test media except PEN.
Main characteristics of these species have been reported to be Gram-negative, non pigmented rods which did not fall into any other group. However, as far as the present study was concerned, no consistent results could be seen for distinguishing the group II organisms from the group III-IV.

Ps. rebescens (IAM 1510) and Ps. sp. 56, one of the isolate were identified to be group III-IV according to the key shown in Table 1, while both Ps. sp. 77 were identified as group II.

However, marked differences could be seen in their responses when the data on group II were compared more in detail with those of group IIIIV, viz., both reference cultures and isolates of group II species grew on either SS agar or STR, while the majority of species belonging to group III-IV failed to grow on the same media.

Achromobacter:

As shown in Tables 4 and 5, among 7 reference cultures and 21 isolates of Achromobacter, only 2 strains were identified to be Achromobacter species by the replica plating method. Of all the test organisms including 7 reference cultures, a wide variety of responses on daughter plates were observed.

According to Corlett et al., ${ }^{5)}$ genus Achromobacter may be identified by their growth on TY, VAN and 110 medium, and negative growth responses on $\mathrm{SS}, \mathrm{S}-\mathrm{C}, \mathrm{NEO}$ were characterized (of Table 1).

Of these characteristics, Corlett et $a l .^{5)}$ 
Table 9. Growth Response of Genus Achromobacter on Corlett's Basal Medium (TPN) Containing Varying Concentration of $\mathrm{NaCl}$

\begin{tabular}{|c|c|c|c|c|c|c|c|c|}
\hline & \multirow{2}{*}{\multicolumn{2}{|c|}{ Name of organisms }} & \multicolumn{6}{|c|}{ Concentration of $\mathrm{NaCl}, \%$} \\
\hline & & & 0 & 1 & 3 & 5 & 7 & 9 \\
\hline 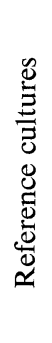 & $\begin{array}{l}\text { Achromobacter } \\
\text { A. sp. } \\
\text { A. guttatus } \\
\text { A. lacticum } \\
\text { A. halophilus } \\
\text { A. stenohalis } \\
\text { A. cycloclastes } \\
\text { A. candicans }\end{array}$ & $\begin{array}{l}\text { NCMB } 29 \\
\text { NCMB } 132 \\
\text { NCMB } 1405 \\
\text { NCIB } 8209 \\
\text { AHU } 1333 \\
\text { AHU } 1336 \\
\text { IAM } 1013\end{array}$ & $\begin{array}{l}- \\
- \\
+ \\
+ \\
- \\
- \\
+ \\
+\end{array}$ & $\begin{array}{l}+ \\
+ \\
+ \\
+ \\
- \\
- \\
+ \\
+\end{array}$ & $\begin{array}{l}+ \\
+ \\
+ \\
+ \\
+ \\
+ \\
- \\
+\end{array}$ & $\begin{array}{l}- \\
+ \\
- \\
- \\
+ \\
+ \\
- \\
-\end{array}$ & $\begin{array}{l}- \\
+ \\
- \\
- \\
+ \\
+ \\
- \\
-\end{array}$ & $\begin{array}{l}- \\
- \\
- \\
- \\
+ \\
+ \\
- \\
-\end{array}$ \\
\hline $\begin{array}{l}0 \\
0 \\
\frac{\pi}{0} \\
0 \\
0\end{array}$ & $\begin{array}{c}\text { Achromobacter } \\
\text { " } \\
\text { " } \\
\text { " } \\
\text { " } \\
\text { " } \\
\text { " } \\
\text { " } \\
\text { " } \\
\text { " } \\
\text { " }\end{array}$ & $\begin{array}{r}58 \\
65 \\
79 \\
80 \\
115 \\
179 \\
185 \\
189 \\
193 \\
195 \\
196 \\
197\end{array}$ & $\begin{array}{l}- \\
- \\
- \\
- \\
+ \\
- \\
- \\
- \\
- \\
- \\
- \\
-\end{array}$ & $\begin{array}{l}+ \\
+ \\
+ \\
+ \\
+ \\
+ \\
- \\
+ \\
+ \\
+ \\
+ \\
+ \\
+\end{array}$ & $\begin{array}{l}+ \\
+ \\
+ \\
+ \\
+ \\
+ \\
+ \\
+ \\
+ \\
+ \\
+ \\
+\end{array}$ & $\begin{array}{l}+ \\
+ \\
+ \\
+ \\
- \\
+ \\
+ \\
+ \\
+ \\
+ \\
+ \\
+\end{array}$ & $\begin{array}{l}+ \\
+ \\
+ \\
+ \\
- \\
+ \\
- \\
+ \\
+ \\
- \\
+ \\
+\end{array}$ & $\begin{array}{l}- \\
- \\
- \\
+ \\
- \\
+ \\
- \\
- \\
- \\
- \\
+ \\
-\end{array}$ \\
\hline & Total of positiv & reaction & 5 & 16 & 19 & 14 & 11 & 5 \\
\hline & $\%$ of positive & eaction & 25 & 80 & 95 & 70 & 55 & 25 \\
\hline
\end{tabular}

claimed that it was the 110 medium which may separate Achromobacter species from the other Gram-negative rods. However, present experiment revealed that none in the 7 reference cultures and only 2 out of 21 isolates did grow on the 110 medium. Among the Achromobacter species, there exist both marine and terrestrial types, so it may hardly be believed that all Achromobacter species can resist to such a high $\mathrm{NaCl}$ concentration as $7.5 \%$ contained in the 110 medium.

As shown in Table 9, growth responses of both reference cultures and isolates of the genus Achromobacter at varying concentrations of $\mathrm{NaCl}$ revealed that 12 test organisms out of 20 grew on the $7 \% \mathrm{NaCl}$ agar, and 6 cultures did on the $9 \%$ $\mathrm{NaCl}$, while the remainders failed to grow on the same plates. In other words, the $\mathrm{NaCl}$ resistant species to $7 \% \mathrm{NaCl}$ in the test organisms was in the ratio of $60 \%(12 / 20)$, and to $9 \% \mathrm{NaCl}$ was $30 \%$ (6/20). From these findings, it may be concluded that the growth response on 110 medium would not be an appropriate key for the identification of genus Achromobacter.

It is to be noted that $25 \%$ of Achromobacter species tested in this experiment could grow at $0 \% \mathrm{NaCl}, 75 \%$ grew at $1 \% \mathrm{NaCl}$ and $90 \%$ at $3 \%$ $\mathrm{NaCl}$. These facts give rise to an important problem on the isolation step of the Corlett's replica plating method. The $\mathrm{NaCl}$ concentration in TPN medium was $0.5 \%$, at which greater part of Achromobacter species would fail to grow.

In this meaning, the use of $3 \% \mathrm{NaCl}$ containing agar medium would be more preferable, especially for supporting the growth of Achromobacter species.

On the other hand, the effect of $3 \% \mathrm{NaCl}$ in the medium on the growth of bacteria other than Archromobacter species has to be clarified, and as shown in Table 10, 5 species of Pseudomonas among 19 test organisms were inhibited for their growth 
Table 10. Growth Response of Genus Pseudomonas on Corlett's Basal Medium (TPN) Containing Varying Concentration of $\mathrm{NaCl}$

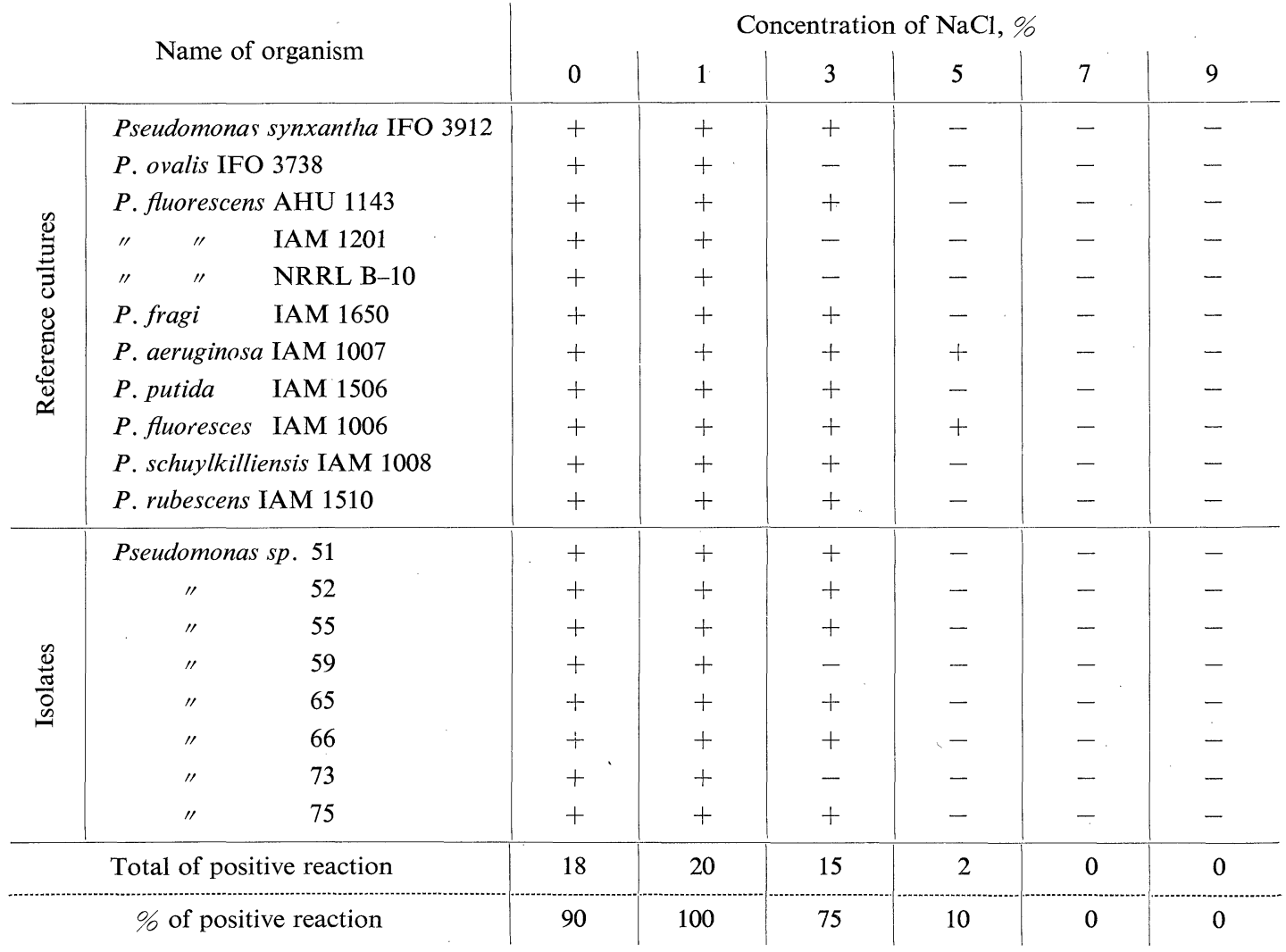

by $3 \%$ of $\mathrm{NaCl}$ in the medium. In order to get more appropriate basal medium for the replica plating, further studies would be needed.

Flavobacterium:

Genus Flavobacterium is characterized by the pigmentation of non-diffusible yellow, red or tan, which may be one of the most important keys for identifying Flavobacterium species (Bergey's Manual). $^{12 !}$

Three reference cultures and 5 isolates of Flavobacterium were identified as Flavobacterium according to the replica plating method. The five isolates used in this study, however, were characterized not only by the insoluble yellow pigmentation but also negative responses to all media so far examined, suggesting that the organisms might belong to Cytophaga rather than Flavobacterium. Hayes $^{13 !}$, reported that the Flavobacterium and Cytophaga had many properties in common, indicating the difficulty in differentiating each other. The genus Cytophaga is included in Flavobacterium in the method according to Corlett et al., ${ }^{5)}$ so, the authors wish to support their opinion from the points of simplicity and convenience in the identification techniques.

As already mentioned, replacement of TPN by King's B medium resulted in enhancement in producing fluorescent pigments by some Pseudomonas group I species. In the next place, effect of King's medium B on the pigmentation of Flavobacterium was examined.

As can be seen in Table 11, of 3 reference cultures of Flavobacterium, a marked difference in the pigmentation was recorded between on TPN and King's medium $\mathrm{B}$, and the pigmentation of test organisms was obviously disturbed when cultured on King's medium.

The difficulty may be easily solved by judging the pigmentation of members of FlavobacteriumCytophaga on the last plate (TPN) in the daughter plate series, or by using an agar plate containing $30 \%$ skim milk (Shewan et $a .^{3 \prime}$ ) in addition to the 
Table 11. Pigmentation of Flavobacterium Reference Cultures on King's Medium B and Corlett's Basal Medium (TPN)

\begin{tabular}{l|c|c|c|c}
\multirow{2}{*}{ Name of organism } & \multicolumn{2}{|c|}{ King's medium B } & \multicolumn{2}{c}{ T P N } \\
& after $24 \mathrm{hrs}$ & after $48 \mathrm{hrs}$ & after 24 hrs & after 48 hrs \\
\hline $\begin{array}{l}\text { F. suaveolens IFO } 3752 \\
\text { F. aquatile IFO } 3772\end{array}$ & white & white & slightly yellow & slightly yellow \\
F. ovalis $\quad$ IFO 1252 & slightly yellow & yellow & slightly yellow & slightly yellow \\
\end{tabular}

Table 12. Coincidence Ratio of the Results Obtained by Corlett's Replica Plating Method to Those of the Method Shown in Fig. 1

\begin{tabular}{|c|c|c|}
\hline Organisms & Reference cultures & Isolates \\
\hline Pseudomonas, group I & $46 \%(6 / 13)$ & $76 \%(186 / 247)$ \\
\hline group II & $100 / /(4 / 4)$ & $99 /(396 / 408)$ \\
\hline group III-IV & $25 \prime \prime(1 / 4)$ & $21 /(8 / 36)$ \\
\hline Achromobacter & $0 \prime \prime(0 / 7)$ & $8 \prime \prime(3 / 38)$ \\
\hline Flavobacterium & $100 / \prime(3 / 3)$ & $100 " /(7 / 7)$ \\
\hline Coliform & $100 / \prime(7 / 7)$ & $100 "(18 / 18)$ \\
\hline
\end{tabular}

daughter plates series.

Coliform:

Seven reference cultures and 4 of the isolates which had been identified as coliform by the method shown in Fig. 1 have also been identified as they were by the replica plating method.

SS medium is primary aimed for differentiating and selecting of Salmonella and Shigella species, aiming to inhibit the Escherichia and Aerobacter species. In this experiment, we found no growth inhibition of coliform on SS medium when replicated, and the primary characteristic of coliform group was their production of a brightpink colony on SS.

The last Table 12 indicates the summarized data on coincidence ratios of the results obtained by Corlett's replica plating method to those determined by the authors' method shown in Fig. 1.

\section{References}

1) E. B. Masurovsky, J. S. Voss, S. A. Goldblith: Appl. Microbiol. 11, 229 (1963).
2) M. S. Hendrie, W. Hodgkiss and J. M. Shewan: Annales de l'Institut Pasteur de Lille 15, 43 (1964).

3) J.M. Shewan, G. Hobbs, W.Hodgkiss: J. Appl. Bacteriol. 23, 379 (1960).

4) N. Kanazas: Appl. Microbiol. 14, 261 (1966).

5) D. A. Corlett, Jr., J. S. Lee, R. O. Sinnhuber: Appl. Microbiol. 13, 808 (1965).

6) D. A. Corlett, Jr., J. S. Lee, R. O. Sinnhuber: Appl. Microbiol. 13, 818 (1965).

7) J. S. Lee, G. C. Wolfe: Food Technol. 21, 841 (1967).

8) J. Lederberg, E. M. Lederberg: J. Bacteriol. 63, 399 (1952).

9) T. Kawabata: Unpublished data

10) Difco Manual, 9th Ed., p. 151 (1953). Difco Laboratories, Detroit, Mich.

11) E. O. King, M. K. Ward, D. E. Raney: J. Lab. \& Clin. Med., 44, 301 (1954).

12) R. S. Breed, E. G. D. Murray, R. R. Smith: Bergey's Manual of Determinative Bacteriology 7th Ed., (1957). The Williams \& Wilkins Co., Baltimore.

13) P. R. Hayes: J. Gen. Microbiol. 30, 1 (1963). 